\title{
Bi-unique range sets with smallest cardinalities for the derivatives of meromorphic functions
}

\author{
Abhijit Banerjee ${ }^{1}$ and Sanjay Mallick ${ }^{2}$ \\ ${ }^{1,2}$ Department of Mathematics, University of Kalyani, West Bengal 741235, India \\ E-mail: abanerjee_kal@yahoo.co.in ${ }^{1}$, abanerjeekal@gmail.com ${ }^{1}$, smallick.ku@gmail.com ${ }^{2}$
}

\begin{abstract}
Inspired by the advent of bi-unique range sets [2], we obtain a new bi-unique range sets, with smallest cardinalities ever for the derivatives of meromorphic functions which improves all the results obtained so far in some sense including a result of Banerjee-Bhattacharjee [4]. Furthermore at the last section we pose an open question for future research.
\end{abstract}

2010 Mathematics Subject Classification. 30D35.

Keywords. Meromorphic function, Uniqueness, Shared set, Weighted sharing.

\section{Introduction, definitions and results}

Let $\overline{\mathbb{C}}=\mathbb{C} \cup\{\infty\}$, where $\mathbb{C}$ denotes the set of all complex numbers. In the paper by any meromorphic function $f$ we always mean it is defined on $\mathbb{C}$. Here we consider standard notations of Nevanlinna theory as explained in [8]. For any non-constant meromorphic function $h(z)$ we define $S(r, h)$ by $S(r, h)=o(T(r, h)),(r \longrightarrow \infty, r \notin E)$ where $E$ denotes any set of positive real number having finite linear measure.

It is well-known to all of us that Gross is the trailblazer of the value sharing problem to the set sharing problem. Hence we have the following definition in the literature.

Definition 1.1. Let for a non constant meromorphic function $f$ and $S \subset \overline{\mathbb{C}}, E_{f}(S)=\bigcup_{a \in S}\{(z, p) \in$ $\mathbb{C} \times \mathbb{N}: f(z)=$ a with multiplicity $p\}\left(\bar{E}_{f}(S)=\bigcup_{a \in S}\{(z, 1) \in \mathbb{C} \times \mathbb{N}: f(z)=a\}\right)$, then we say $f$, $g$ share the set $S \mathrm{CM}(\mathrm{IM})$ if $E_{f}(S)=E_{g}(S)\left(\bar{E}_{f}(S)=\bar{E}_{g}(S)\right)$.

In 2001 Lahiri ([10], [11]) introduced the following notion of scalings between CM and IM which further add essence to the uniqueness literature.

Definition 1.2 ([10], [11]). Let $\mathrm{k}$ be a nonnegative integer or infinity. For $a \in \overline{\mathbb{C}}$ we denote by $E_{k}(a ; f)$ the set of all a-points of f, where an $a$-point of multiplicity $m$ is counted $m$ times if $m \leq k$ and $k+1$ times if $m>k$. If $E_{k}(a ; f)=E_{k}(a ; g)$, we say that $f, g$ share the value a with weight $k$.

We write $f, g$ share $(a, k)$ to mean that $f, g$ share the value a with weight $k$. Clearly if $f, g$ share $(a, k)$ then $f, g$ share $(a, p)$ for any integer $p, 0 \leq p<k$. Also we note that $f, g$ share a value a IM or CM if and only if $f, g$ share $(a, 0)$ or $(a, \infty)$ respectively.

Definition 1.3. [10] For $S \subset \overline{\mathbb{C}}$ we define $E_{f}(S, k)=\cup_{a \in S} E_{k}(a ; f)$, where k is a non-negative integer $a \in S$ or infinity. Clearly $E_{f}(S)=E_{f}(S, \infty)$ and $\bar{E}_{f}(S)=E_{f}(S, 0)$

In 1977, Gross [7] posed his famous question related to the uniqueness of entire functions sharing sets. In connection to that the following question regarding the uniqueness of meromorphic functions was asked.

Tbilisi Mathematical Journal 9(2) (2016), pp. 1-13.

Tbilisi Centre for Mathematical Sciences.

Received by the editors: 29 October 2015.

Accepted for publication: 20 June 2016. 
Question A ([18], [19]). Can one find two finite sets $S_{j}(j=1,2)$ such that any two non-constant meromorphic functions $f$ and $g$ satisfying $E_{f}\left(S_{j}, \infty\right)=E_{g}\left(S_{j}, \infty\right)$ for $j=1,2$ must be identical ?

Germane to the Question A, in $1996 \mathrm{Li}$-Yang[14] provided $S_{1}$ with 1 element and $S_{2}$ with 15 elements such that any two non-constant meromorphic functions $f$ and $g$ satisfying $E_{f}\left(S_{j}\right)=E_{g}\left(S_{j}\right)$ for $j=1,2$ must be identical.

Later on Fang-Guo [6] improved the above result introducing such two sets where $S_{1}$ contains 1 element and $S_{2}$ contains 9 elements.

Lastly in $2002 \mathrm{Yi}$ [18] improved all these results introducing $S_{1}$ with 1 element and $S_{2}$ with 8 elements.

Recently the present first author [1] improved the result of Yi [18] by relaxing the nature of sharing the range sets under the aegis of weighted sharing. He established that there exist two finite sets $S_{1}$ containing 1 element and $S_{2}$ containing 8 elements such that any two non-constant meromorphic functions $f$ and $g$ satisfying $E_{f}\left(S_{1}, 0\right)=E_{g}\left(S_{1}, 0\right)$ and $E_{f}\left(S_{2}, 2\right)=E_{g}\left(S_{2}, 2\right)$ must be identical.

Later on in order to reduce the cardinality of $S_{2}$ the research in this particular set up has somehow been shifted to-wards considering the derivatives of meromorphic functions sharing one or two sets. Below we are recalling some results.

Theorem A. [19] Let $S_{1}=\{\infty\}$ and $S_{2}=\left\{z: z^{n}+a z^{n-1}+b=0\right\}$, where $a, b$ are nonzero constants such that $z^{n}+a z^{n-1}+b=0$ has no repeated root and $n(\geq 7), k$ be two positive integers. Let $f$ and $g$ be two non-constant meromorphic functions such that $E_{f}\left(S_{1}\right)=E_{g}\left(S_{1}\right)$ and $E_{f^{(k)}}\left(S_{2}\right)=E_{g^{(k)}}\left(S_{2}\right)$ then $f^{(k)} \equiv g^{(k)}$.

In 2010, Banerjee-Bhattacharjee [3] proved the following two theorems which improved the above results.

Theorem B. [3] Let $S_{i}, i=1,2$ and $k$ be given as in Theorem $A$. Let $f$ and $g$ be two nonconstant meromorphic functions such that $E_{f}\left(S_{1}, 1\right)=E_{g}\left(S_{1}, 1\right)$ and $E_{f^{(k)}}\left(S_{2}, 2\right)=E_{g^{(k)}}\left(S_{2}, 2\right)$ then $f^{(k)} \equiv g^{(k)}$.

Theorem C. [3] Let $S_{i}, i=1,2$ be given as in Theorem $A$. Let $f$ and $g$ be two non-constant meromorphic functions such that $E_{f}\left(S_{1}, 0\right)=E_{g}\left(S_{1}, 0\right)$ and $E_{f^{(k)}}\left(S_{2}, 3\right)=E_{g^{(k)}}\left(S_{2}, 3\right)$ then $f^{(k)} \equiv$ $g^{(k)}$.

In 2011, Banerjee-Bhattacharjee [4] further improved the above results in the following manner.

Theorem D. [4] Let $S_{i}, i=1,2$ and $k$ be given as in Theorem $A$. Let $f$ and $g$ be two nonconstant meromorphic functions such that $E_{f}\left(S_{1}, 0\right)=E_{g}\left(S_{1}, 0\right)$ and $E_{f^{(k)}}\left(S_{2}, 2\right)=E_{g^{(k)}}\left(S_{2}, 2\right)$ then $f^{(k)} \equiv g^{(k)}$.

Observe that in Theorems $A$-D, one set contains $n$ elements where as the other set contains only $\infty$ and then the authors tried to reduce the value of $n$ as much as possible.

Under such circumstances, patently the following question steps into the literature.

Question B. Is it possible to obtain better result for Question A considering two sets in $\mathbb{C}$ ?

In this perspective the introduction of $\mathrm{Bi}$-Unique range sets can be thought of as the inception of a new direction in set sharing problem. Below we recall the definition. 
Definition 1.4. [2] A pair of finite sets $S_{1}$ and $S_{2}$ in $\mathbb{C}$ is called bi unique range sets for meromorphic (entire) functions with weights $m, k$ if for any two non-constant meromorphic (entire) functions $f$ and $g, E_{f}\left(S_{1}, m\right)=E_{g}\left(S_{1}, m\right), E_{f}\left(S_{2}, k\right)=E_{g}\left(S_{2}, k\right)$ implies $f \equiv g$. We write $S_{i}$ 's $i=1,2$ as BURSM $m, k$ (BURSEm, $k$ ) in short. As usual if both $m=k=\infty$, we say $S_{i}$ 's $i=1,2$ as BURSM (BURSE).

In apt to this we recall the following theorem of H.X.Yi [17] which is most probably the first BURSM prior to its introduction.

Theorem E. [17] Let $S_{1}=\left\{a+b, a+b \omega, \ldots, a+b \omega^{n-1}\right\}, S_{2}=\left\{c_{1}, c_{2}\right\}$ where $\omega=e^{\frac{2 \pi i}{n}}$ and $b \neq 0$, $c_{1} \neq a, c_{2} \neq a,\left(c_{1}-a\right)^{n} \neq\left(c_{2}-a\right)^{n},\left(c_{k}-a\right)^{n}\left(c_{j}-a\right)^{n} \neq b^{2 n}(k, j=1,2)$ are constants. If $n \geq 9$ then Then $S_{i}$ 's $i=1,2$ are BURSM.

Afterwards in $2012 \mathrm{Yi}$ and $\mathrm{Li}$ [13] improved the above theorem providing the following result.

Theorem F. [16] Let $S_{1}=\{0,1\}, S_{2}=\left\{z: \frac{(n-1)(n-2)}{2} z^{n}-n(n-2) z^{n-1}+\frac{n(n-1)}{2} z^{n-2}+1=0\right\}$, where $n(\geq 5)$ is an integer. Then $S_{i}$ 's $i=1,2$ are $B U R S M$.

The above result is obviously better than all the results discussed so far in the direction of Question A. So Theorem F provides the affirmative answer of Question B and enriches the notion of BURSM.

Observe that the set $S_{1}$ in Theorem $F$ is nothing but the set of zeros of the derivatives of the polynomial whose zeros are used to form the set $S_{2}$. With the help of this inherited property the first author tried to generalize the polynomial used to form $S_{2}$ of Theorem $F$ and obtain the following result.

Theorem G. [2] Let $S_{1}=\{0,1\}, S_{2}=\left\{z: \frac{(n-1)(n-2)}{2} z^{n}-n(n-2) z^{n-1}+\frac{n(n-1)}{2} z^{n-2}-c=0\right\}$, where $n(\geq 5)$ is an integer and $c \neq 0,1, \frac{1}{2}$ is a complex number such that $c^{2}-c+1 \neq 0$. Then $S_{i}$ 's $i=1,2$ are $B U R S M 1,3, B U R S M 3,2$.

Clearly Theorem $G$ directly improves Theorem $F$. Notice that the polynomial used in Theorems $F-G$ are of the same type. Recently to-wards finding different BURSM, present authors proved the following theorem with a different type of polynomial.

Theorem H. [5] Let $S_{1}=\left\{0, c_{1}, c_{2}\right\}, S_{2}=\left\{z: z^{5}+a z^{3}+b=0\right\}$ where $a$ and $b$ be two nonzero constants such that $z^{5}+a z^{3}+b=0$ has no multiple root. If $E_{f}\left(S_{1}, p\right)=E_{g}\left(S_{1}, p\right)$, and $E_{f}\left(S_{2}, m\right)=$ $E_{g}\left(S_{2}, m\right)$, with $2 p(4 m-9)>15$, then $f \equiv g$.

If we minutely delve into the construction of BURSM's used in Theorems $F-H$ then we see that the underlying polynomial whose zeros are forming $S_{2}$ is the backbone of a BURSM and $S_{1}$ is the collection of all the zeros of derivative of the polynomial whose zeros generate $S_{2}$. Also we note that in Theorems $F$ - $H$ the cardinality of the second set could not further be diminished rather for the variation of the polynomial corresponding to $S_{2}$ the cardinality of $S_{1}$ increases even if the cardinality of $S_{2}$ remains the same. Naturally the following two questions comes in mind in terms of BURSM concerning the improvements of all the above results.

Question 1.1. Is it possible to further reduce the cardinality as well as relax the nature of sharing the set $S_{2}$ ? 
Question 1.2. Is there any compulsion to consider all the zeros of derivative of the underlying polynomial to form $S_{1}$ ?

In this paper we shall show that if we consider the derivatives of the meromorphic function instead of the original function as used in Theorems $A-D$ then we can answer Question 1.1 and 1.2. We have the next theorem as the main result of this paper which is also the best result ever obtained till today in terms of BURSM for a special class of meromorphic function. Henceforth throughout the paper for an integer $n$ and a non-zero constant $a$, let us denote $-a \frac{n-1}{n}$ by $c_{1}$.

Theorem 1.1. Let $S_{1}=\{0\}, S_{2}=\left\{z: z^{n}+a z^{n-1}+b=0\right\}$, where $n(\geq 4)$ be an integer and $a$ and $b$ be two nonzero constants such that $z^{n}+a z^{n-1}+b$ has no multiple zero. If for two non constant meromorphic functions $f$ and $g$, with $f^{(k)}$ and $g^{(k)}$ having no simple $c_{1}$ points; $E_{f^{(k)}}\left(S_{1}, 1\right)=E_{g^{(k)}}\left(S_{1}, 1\right)$ and $E_{f^{(k)}}\left(S_{2}, 2\right)=E_{g^{(k)}}\left(S_{2}, 2\right)$, then $f^{(k)} \equiv g^{(k)}$.

The following example shows that in Theorem $1.1 a \neq 0$ is necessary.

Example 1.1. Let $f(z)=\sqrt[4]{-b} e^{z}$ and $g(z)=(-1)^{k} \sqrt[4]{-b} e^{-z}$ and $S_{1}=\{0\}, S_{2}=\left\{z: z^{4}+b=0\right\}$. Then $f^{(k)}, g^{(k)}$ share $\left(S_{i}, \infty\right), i=1,2$ but $f^{(k)} \not \equiv g^{(k)}$.

The next example shows that $S_{2}$ of Theorem 1.1 can not be replaced by any arbitrary set containing 4 elements.

Example 1.2. Let $S_{1}=\{0\}$ and $S_{2}=\{i,-1,-i, 1\}$. Then for the functions $f=i e^{z}$ and $g=-e^{z}$ we have $f^{(k)}, g^{(k)}$ share $\left(S_{i}, \infty\right), i=1,2$ but $f^{(k)} \not \equiv g^{(k)}$.

Though for the standard definitions and notations of the value distribution theory we refer to [8], we now explain some notations which are frequently used in the paper.

Definition 1.5. [9] For $a \in \mathbb{C} \cup\{\infty\}$ we denote by $N(r, a ; f \mid=1)$ the counting function of simple $a$ points of $f$. For a positive integer $m$ we denote by $N(r, a ; f \mid \leq m)(N(r, a ; f \mid \geq m))$ the counting function of those $a$ points of $f$ whose multiplicities are not greater(less) than $m$ where each $a$ point is counted according to its multiplicity.

$\bar{N}(r, a ; f \mid \leq m)(\bar{N}(r, a ; f \mid \geq m))$ are defined similarly, where in counting the $a$-points of $f$ we ignore the multiplicities.

Also $N(r, a ; f \mid<m), N(r, a ; f \mid>m), \bar{N}(r, a ; f \mid<m)$ and $\bar{N}(r, a ; f \mid>m)$ are defined analogously.

Definition 1.6 ([10], [11]). Let $f, g$ share a value $a \mathrm{IM}$. We denote by $\bar{N}_{*}(r, a ; f, g)$ the reduced counting function of those $a$-points of $f$ whose multiplicities differ from the multiplicities of the corresponding $a$-points of $g$. Clearly $\bar{N}_{*}(r, a ; f, g) \equiv \bar{N}_{*}(r, a ; g, f)$ and in particular if $f$ and $g$ share $(a, p)$ then $\bar{N}_{*}(r, a ; f, g) \leq \bar{N}(r, a ; f \mid \geq p+1)=\bar{N}(r, a ; g \mid \geq p+1)$.

Definition 1.7. Let $a, b_{1}, b_{2}, \ldots, b_{q} \in \mathbb{C} \cup\{\infty\}$. We denote by $N\left(r, a ; f \mid g \neq b_{1}, b_{2}, \ldots, b_{q}\right)$ the counting function of those $a$-points of $f$, counted according to multiplicity, which are not the $b_{i}$-points of $g$ for $i=1,2, \ldots, q$. 


\section{Lemmas}

In this section we present some lemmas which will be needed in the sequel. Let $F$ and $G$ be two non-constant meromorphic functions defined in $\mathbb{C}$ as follows.

$$
F=\frac{P\left(f^{(k)}\right)}{-b}=\frac{\left(f^{(k)}\right)^{n-1}\left(f^{(k)}+a\right)}{-b}, \quad G=\frac{P\left(g^{(k)}\right)}{-b}=\frac{\left(g^{(k)}\right)^{n-1}\left(g^{(k)}+a\right)}{-b},
$$

where $n(\geq 2)$ and $k$ are two positive integers and for a meromorphic function $h$ we put $P(h)=(h)^{n}+a(h)^{n-1}$. Henceforth we shall denote by $H$ and $\Phi$ the following two functions

$$
H=\left(\frac{F^{\prime \prime}}{F^{\prime}}-\frac{2 F^{\prime}}{F-1}\right)-\left(\frac{G^{\prime \prime}}{G^{\prime}}-\frac{2 G^{\prime}}{G-1}\right)
$$

and

$$
\Phi=\frac{F^{\prime}}{F-1}-\frac{G^{\prime}}{G-1} .
$$

Lemma 2.1. ([11], Lemma 1) Let $F, G$ be two non-constant meromorphic functions sharing $(1,1)$ and $H \not \equiv 0$. Then

$$
N(r, 1 ; F \mid=1)=N(r, 1 ; G \mid=1) \leq N(r, H)+S(r, F)+S(r, G) .
$$

Lemma 2.2. Let $S_{1}$ and $S_{2}$ be defined as in Theorem 1.1 and $F, G$ be given by (2.1). If for two nonconstant meromorphic functions $f$ and $g, E_{f^{(k)}}\left(S_{1}, p\right)=E_{g^{(k)}}\left(S_{1}, p\right), E_{f^{(k)}}\left(S_{2}, 0\right)=E_{g^{(k)}}\left(S_{2}, 0\right)$, where $0 \leq p<\infty$ and $H \not \equiv 0$ then

$$
\begin{aligned}
N(r, H) \leq & \bar{N}\left(r, 0 ; f^{(k)} \mid \geq p+1\right)+\bar{N}(r, \infty ; f)+\bar{N}(r, \infty ; g) \\
& +\bar{N}_{*}(r, 1 ; F, G)+\bar{N}_{0}\left(r, 0 ; F^{\prime}\right)+\bar{N}_{0}\left(r, 0 ; G^{\prime}\right),
\end{aligned}
$$

where $\bar{N}_{0}\left(r, 0 ; F^{\prime}\right)$ is the reduced counting function of those zeros of $F^{\prime}$ which are not the zeros of $f^{(k)}(F-1)$ and $\bar{N}_{0}\left(r, 0 ; G^{\prime}\right)$ is similarly defined.

Proof. We note that $F^{\prime}=\frac{\left(f^{(k)}\right)^{n-2}\left(n f^{(k)}+a(n-1)\right) f^{(k+1)}}{-b}, G^{\prime}=\frac{\left(f^{(k)}\right)^{n-2}\left(n g^{(k)}+a(n-1)\right) g^{(k+1)}}{-b}$ and

$$
\begin{aligned}
& F^{\prime \prime}=\frac{\left(f^{(k)}\right)^{n-2}\left(n f^{(k)}+a(n-1)\right) f^{(k+2)}+\left(f^{(k)}\right)^{n-3}\left(n(n-1) f^{(k)}+a(n-1)(n-2)\right)\left(f^{(k+1)}\right)^{2}}{-b}, \\
& G^{\prime \prime}=\frac{\left(g^{(k)}\right)^{n-2}\left(n g^{(k)}+a(n-1)\right) g^{(k+2)}+\left(g^{(k)}\right)^{n-3}\left(n(n-1) g^{(k)}+a(n-1)(n-2)\right)\left(g^{(k+1)}\right)^{2}}{-b} .
\end{aligned}
$$

So

$$
\begin{aligned}
H=\quad & \frac{(n-1)\left(n f^{(k)}+a(n-2)\right) f^{(k+1)}}{f^{(k)}\left(n f^{(k)}+a(n-1)\right)}-\frac{(n-1)\left(n g^{(k)}+a(n-2)\right) g^{(k+1)}}{g^{(k)}\left(n g^{(k)}+a(n-1)\right)} \\
& +\frac{f^{(k+2)}}{f^{(k+1)}}-\frac{g^{(k+2)}}{g^{(k+1)}}-\left(\frac{2 F^{\prime}}{F-1}-\frac{2 G^{\prime}}{G-1}\right) .
\end{aligned}
$$

Clearly $F$ and $G$ share $(1,0)$. Since $H$ has only simple poles, the lemma can easily be proved by simple calculation. 
Lemma 2.3. [4] Let $f$ and $g$ be two meromorphic functions sharing $(1, m)$, where $1 \leq m<\infty$. Then

$$
\bar{N}(r, 1 ; f)+\bar{N}(r, 1 ; g)-N(r, 1 ; f \mid=1)+\left(m-\frac{1}{2}\right) \bar{N}_{*}(r, 1 ; f, g) \leq \frac{1}{2}[N(r, 1 ; f)+N(r, 1 ; g)] .
$$

Lemma 2.4. [15] Let $f$ be a non-constant meromorphic function and let

$$
R(f)=\frac{\sum_{k=0}^{n} a_{k} f^{k}}{\sum_{j=0}^{m} b_{j} f^{j}}
$$

be an irreducible rational function in $f$ with constant coefficients $\left\{a_{k}\right\}$ and $\left\{b_{j}\right\}$ where $a_{n} \neq 0$ and $b_{m} \neq 0$ Then

$$
T(r, R(f))=d T(r, f)+S(r, f)
$$

where $d=\max \{n, m\}$.

Lemma 2.5. Let $S_{1}$ and $S_{2}$ be defined as in Theorem 1.1 and $F, G$ be given by (2.1). If for two nonconstant meromorphic functions $f$ and $g, E_{f^{(k)}}\left(S_{1}, p\right)=E_{g^{(k)}}\left(S_{1}, p\right), E_{f^{(k)}}\left(S_{2}, m\right)=E_{g^{(k)}}\left(S_{2}, m\right)$, $0 \leq p<\infty$ and $\Phi \not \equiv 0$ then

$$
\begin{aligned}
& (3 p+2)\left\{\bar{N}\left(r, 0 ; f^{(k)} \mid \geq p+1\right)\right\} \\
\leq & \bar{N}(r, \infty ; f)+\bar{N}(r, \infty ; g)+\bar{N}_{*}(r, 1 ; F, G)+S\left(r, f^{(k)}\right)+S\left(r, g^{(k)}\right) .
\end{aligned}
$$

Proof. By the given condition clearly $F$ and $G$ share $(1, m)$. Also we see that

$$
\Phi=\frac{\left(f^{(k)}\right)^{n-2}\left(n f^{(k)}+a(n-1)\right) f^{(k+1)}}{-b(F-1)}-\frac{\left(g^{(k)}\right)^{n-2}\left(n g^{(k)}+a(n-1)\right) g^{(k+1)}}{-b(G-1)} .
$$

Let $z_{0}$ be a zero of $f^{(k)}$ with multiplicity $r$. Since $E_{f^{(k)}}\left(S_{1}, p\right)=E_{g^{(k)}}\left(S_{1}, p\right)$ then that would be a zero of $\Phi$ of multiplicity $(n-2) r+r-1$ i.e., of multiplicity $(n-1) r-1$ if $r \leq p$ and a zero of multiplicity at least $(n-2)(p+1)+p$ i.e., a zero of multiplicity at least $(n-1) p+(n-2) \geq 3 p+2$ if $r>p$. So by a simple calculation we can write

$$
\begin{aligned}
& \{3 p+2\}\left\{\bar{N}\left(r, 0 ; f^{(k)} \mid \geq p+1\right)\right\} \\
\leq & N(r, 0 ; \Phi) \\
\leq & T(r, \Phi) \\
\leq & N(r, \infty ; \Phi)+S(r, F)+S(r, G) \\
\leq & \bar{N}_{*}(r, 1 ; F, G)+\bar{N}(r, \infty ; f)+\bar{N}(r, \infty ; g)+S\left(r, f^{(k)}\right)+S\left(r, g^{(k)}\right) .
\end{aligned}
$$

Q.E.D.

Lemma 2.6. Let $S_{1}, S_{2}$ be defined as in Theorem 1.1 and $F, G$ be given by (2.1). If for two non-constant meromorphic functions $f$ and $g$, with $f^{(k)}$ and $g^{(k)}$ having no simple $c_{1}$ points; 
$E_{f^{(k)}}\left(S_{1}, p\right)=E_{g^{(k)}}\left(S_{1}, p\right), E_{f^{(k)}}\left(S_{2}, m\right)=E_{g^{(k)}}\left(S_{2}, m\right)$, where $0 \leq p<\infty, 2 \leq m<\infty$ and $H \not \equiv 0$, then

$$
\begin{aligned}
& n\left\{T\left(r, f^{(k)}\right)+T\left(r, g^{(k)}\right\}\right. \\
\leq & 2 \bar{N}\left(r, 0 ; f^{(k)}\right)+\bar{N}\left(r, 0 ; f^{(k)} \mid \geq p+1\right)+2\{\bar{N}(r, \infty ; f)+\bar{N}(r, \infty ; g)\} \\
& +\frac{1}{2}[N(r, 1 ; F)+N(r, 1 ; G)]-\left(m-\frac{3}{2}\right) \bar{N}_{*}(r, 1 ; F, G)+S\left(r, f^{(k)}\right)+S\left(r, g^{(k)}\right) .
\end{aligned}
$$

Proof. By the second fundamental theorem we get

$$
\begin{aligned}
& n\left\{T\left(r, f^{(k)}\right)+T\left(r, g^{(k)}\right)\right\} \\
\leq & \bar{N}(r, 1 ; F)+\bar{N}\left(r, 0 ; f^{(k)}\right)+\bar{N}(r, \infty ; f)+\bar{N}(r, 1 ; G) \\
& +\bar{N}\left(r, 0 ; g^{(k)}+\bar{N}(r, \infty ; g)-N_{0}\left(r, 0 ; f^{(k+1)}\right)\right. \\
& -N_{0}\left(r, 0 ; g^{(k+1)}\right)+S\left(r, f^{(k)}\right)+S\left(r, g^{(k)}\right) .
\end{aligned}
$$

Using Lemmas 2.1, 2.2, 2.3 and 2.4 we note that

$$
\begin{aligned}
& \bar{N}(r, 1 ; F)+\bar{N}(r, 1 ; G) \\
\leq & \frac{1}{2}[N(r, 1 ; F)+N(r, 1 ; G)]+N(r, 1 ; F \mid=1)-\left(m-\frac{1}{2}\right) \bar{N}_{*}(r, 1 ; F, G) \\
\leq & \frac{1}{2}[N(r, 1 ; F)+N(r, 1 ; G)]+\bar{N}\left(r, 0 ; f^{(k)} \mid \geq p+1\right) \\
& +\bar{N}(r, \infty ; f)+\bar{N}(r, \infty ; g)-\left(m-\frac{3}{2}\right) \bar{N}_{*}(r, 1 ; F, G)+\bar{N}_{0}\left(r, 0 ; F^{\prime}\right)+\bar{N}_{0}\left(r, 0 ; G^{\prime}\right) \\
& +S\left(r, f^{(k)}\right)+S\left(r, g^{(k)}\right) .
\end{aligned}
$$

Using (2.5) in (2.4) and noting that $N\left(r, c_{1} ; f^{(k)} \mid=1\right)=S\left(r, f^{(k)}\right), \bar{N}\left(r, c_{1} ; g^{(k)} \mid=1\right)=S\left(r, g^{(k)}\right)$ and $\bar{N}\left(r, 0 ; f^{(k)}\right)=\bar{N}\left(r, 0 ; g^{(k)}\right)$, the lemma follows.

Q.E.D.

Lemma 2.7. Let $f, g$ be two non-constant meromorphic functions such that $E_{f^{(k)}}\left(S_{1}, 0\right)=E_{g^{(k)}}\left(S_{1}, 0\right)$. Then $\left(f^{(k)}\right)^{n-1}\left(f^{(k)}+a\right) \equiv\left(g^{(k)}\right)^{n-1}\left(g^{(k)}+a\right)$ implies $f^{(k)} \equiv g^{(k)}$, where $n(\geq 2)$ is an integer, $k$ is a positive integer and $a$ is a nonzero finite constant.

Proof. Since $E_{f^{(k)}}\left(S_{1}, 0\right)=E_{g^{(k)}}\left(S_{1}, 0\right)$ and

$$
\left(f^{(k)}\right)^{n-1}\left(f^{(k)}+a\right) \equiv\left(g^{(k)}\right)^{n-1}\left(g^{(k)}+a\right) .
$$

Therefore clearly from $(2.6)$ we conclude that $f^{(k)}$ and $g^{(k)}$ share $(0, \infty)$ and $(\infty, \infty)$. We also note that $\Theta\left(\infty ; f^{(k)}\right)+\Theta\left(\infty ; g^{(k)}\right) \geq 2-\frac{2}{k+1}=\frac{2 k}{k+1}>0$. Now the lemma can be proved in the line of proof of Lemma 3 [13].

Q.E.D.

Lemma 2.8. Let $S_{1}, S_{2}$ be defined as in Theorem 1.1. If for two non-constant meromorphic function $f$ and $g, E_{f^{(k)}}\left(S_{1}, 0\right)=E_{g^{(k)}}\left(S_{1}, 0\right), E_{f^{(k)}}\left(S_{2}, m\right)=E_{g^{(k)}}\left(S_{2}, m\right)$ where $2 \leq m<\infty$ and $\Phi \not \equiv 0$. Also let $\omega_{1}, \omega_{2} \ldots \omega_{n}$ are the members of the set $S_{2}$. Then

$$
\bar{N}_{*}(r, 1 ; F, G) \leq \frac{2}{2 m-1}[\bar{N}(r, \infty ; f)+\bar{N}(r, \infty ; g)]+S\left(r, f^{(k)}\right)+S\left(r, g^{(k)}\right)
$$


Proof. First we note that ' 0 ' is not a member of $S_{2}$. Therefore proceeding as follows with the help of Lemma 2.5 for $p=0$ we get,

$$
\begin{aligned}
& \bar{N}_{*}(r, 1 ; F, G) \\
\leq & \bar{N}(r, 1 ; F \mid \geq m+1) \\
\leq & \frac{1}{m}(N(r, 1 ; F)-\bar{N}(r, 1 ; F)) \\
\leq & \frac{1}{m}\left[\sum_{j=1}^{n}\left(N\left(r, \omega_{j} ; f^{(k)}\right)-\bar{N}\left(r, \omega_{j} ; f^{(k)}\right)\right)\right] \\
\leq & \frac{1}{m}\left[N\left(r, 0 ; f^{(k+1)} \mid f^{(k)} \neq 0\right)\right] \\
\leq & \frac{1}{m}\left[N\left(r, \infty ; \frac{f^{(k)}}{f^{(k+1)}}\right)\right] \\
\leq & \frac{1}{m}\left[N\left(r, \infty ; \frac{f^{(k+1)}}{f^{(k)}}\right)\right]+S\left(r, f^{(k)}\right) \\
\leq & \frac{1}{m}\left[\bar{N}\left(r, 0 ; f^{(k)}\right)+\bar{N}(r, \infty ; f)\right]+S\left(r, f^{(k)}\right) \\
\leq & \frac{1}{2 m}\left[3 \bar{N}(r, \infty ; f)+\bar{N}(r, \infty ; g)+\bar{N}_{*}(r, 1 ; F, G)\right]+S\left(r, f^{(k)}\right)+S\left(r, g^{(k)}\right),
\end{aligned}
$$

which clearly implies

$$
\bar{N}_{*}(r, 1 ; F, G) \leq \frac{1}{2 m-1}[3 \bar{N}(r, \infty ; f)+\bar{N}(r, \infty ; g)]+S\left(r, f^{(k)}\right)+S\left(r, g^{(k)}\right) .
$$

Similarly, applying the above method for $G$ instead of $F$ we can obtain

$$
\bar{N}_{*}(r, 1 ; F, G) \leq \frac{1}{2 m-1}[3 \bar{N}(r, \infty ; g)+\bar{N}(r, \infty ; f)]+S\left(r, f^{(k)}\right)+S\left(r, g^{(k)}\right) .
$$

Now adding (2.7) and (2.8) we get the desired result.

Q.E.D.

\section{Proof of the theorem}

Proof of Theorem 1.1. Let $F, G$ be given by (2.1). Then $F$ and $G$ share $(1,3)$. We consider the following cases.

Case 1. Suppose that $\Phi \not \equiv 0$

Subcase 1.1. Let $H \not \equiv 0$. Then using Lemma 2.6 for $m=2$, Lemma 2.5 for $p=0$ and $p=1$, 
Lemma 2.8 for $m=2$ and Lemma 2.4 we obtain,

$$
\begin{aligned}
& n\left\{T\left(r, f^{(k)}\right)+T\left(r, g^{(k)}\right)\right\} \\
\leq & 2 \bar{N}\left(r, 0 ; f^{(k)}\right)+\bar{N}\left(r, 0 ; f^{(k)} \mid \geq 2\right)+2\{\bar{N}(r, \infty ; f)+\bar{N}(r, \infty ; g)\}-\frac{1}{2} \bar{N}_{*}(r, 1 ; F, G) \\
& +\frac{1}{2}[N(r, 1 ; F)+N(r, 1 ; G)]+S\left(r, f^{(k)}\right)+S\left(r, g^{(k)}\right) \\
\leq & \left\{1+\frac{1}{5}+2\right\}\{\bar{N}(r, \infty ; f)+\bar{N}(r, \infty ; g)\}+\left\{1+\frac{1}{5}-\frac{1}{2}\right\} \bar{N}_{*}(r, 1 ; F, G) \\
& +\frac{1}{2}[N(r, 1 ; F)+N(r, 1 ; G)]+S\left(r, f^{(k)}\right)+S\left(r, g^{(k)}\right) \\
\leq & \left\{\frac{16}{5}+\frac{14}{30}\right\}\{\bar{N}(r, \infty ; f)+\bar{N}(r, \infty ; g)\}+\frac{1}{2}[N(r, 1 ; F)+N(r, 1 ; G)]+S\left(r, f^{(k)}\right)+S\left(r, g^{(k)}\right) \\
\leq & \left\{\frac{n}{2}+\frac{11}{3(k+1)}\right\}\left[T\left(r, f^{(k)}\right)+T\left(r, g^{(k)}\right)\right]+S\left(r, f^{(k)}\right)+S\left(r, g^{(k)}\right),
\end{aligned}
$$

which gives a contradiction for $n \geq 4$.

Subcase 1.2 Let $H \equiv 0$. Then

$$
\frac{1}{F-1} \equiv \frac{A}{G-1}+B
$$

where $A(\neq 0), B$ are constants. Also $T(r, F)=T(r, G)+O(1)$. i.e.,

$$
n T\left(r, f^{(k)}\right)=n T\left(r, g^{(k)}\right)+O(1) .
$$

We now consider the following cases.

\section{Subcase 1.2.1.}

Let $B=0$. From (3.1) we get

$$
\frac{1}{F-1} \equiv \frac{A}{G-1}
$$

i.e.,

$$
G^{\prime} \equiv A F^{\prime}
$$

i.e.,

$$
\Phi \equiv 0,
$$

a contradiction.

Subcase 1.2.2.

If $B \neq 0$ then

$$
F-1 \equiv \frac{G-1}{B G+A-B}
$$

Subcase 1.2.2.1.

If $A-B \neq 0$, then from (3.3) we get

$$
F-1 \equiv \frac{G-1}{B\left(G-\left(\frac{B-A}{B}\right)\right)} .
$$


Subcase 1.2.2.1.1.

If $g^{(k)}-c_{1}$ is a repeated factor of $G-\frac{B-A}{B}$ then

$$
\left(g^{(k)}-c_{1}\right)^{2} \prod_{i=1}^{n-2}\left(g^{(k)}-\alpha_{i}\right) \equiv \frac{1}{B} \frac{G-1}{F-1},
$$

where $g^{(k)}-\alpha_{i}$ 's $(i=1,2, \ldots, n-2)$ are the distinct simple factors of $G-\frac{B-A}{B}$. Since $\frac{B-A}{B} \neq 1$ therefore $c_{1}$ points and $\alpha_{i}$ points of $g^{(k)}$ are neutralised by the poles of $f$. Now if $z_{0}$ is a zero of $g^{(k)}-c_{1}$ of multiplicity $p$, then it would be pole of $f^{(k)}$ of multiplicity $q$ such that $2 p=n q \geq n(k+1)$. Similarly for a zero of $g^{(k)}-\alpha_{i}$ of multiplicity $r$ is a pole of $f^{(k)}$ of multiplicity $s$ (say) we have $r=n s \geq n(k+1)$. So in view of the second fundamental theorem and (3.2) we get

$$
(n-2) T\left(r, g^{(k)}\right) \leq \sum_{i=1}^{n-2} \bar{N}\left(r, \alpha_{i} ; g^{(k)}\right)+\bar{N}\left(r, c_{1} ; g^{(k)}\right)+\bar{N}(r, \infty ; g)+S\left(r, g^{(k)}\right)
$$

i.e.,

$$
(n-2) T\left(r, g^{(k)}\right) \leq \frac{(n-2)}{n(k+1)} T\left(r, g^{(k)}\right)+\frac{2}{n(k+1)} T\left(r, g^{(k)}\right)+\frac{1}{k+1} T\left(r, g^{(k)}\right)+S\left(r, g^{(k)}\right),
$$

which gives a contradiction for $n \geq 4$.

Subcase 1.2 .2 .1 .2 . If $\left(g^{(k)}-c_{1}\right)$ is not a factor of $G-\frac{B-A}{B}$ then

$$
\prod_{i=1}^{n}\left(g^{(k)}-\beta_{i}\right) \equiv \frac{1}{B} \frac{G-1}{F-1},
$$

where $g^{(k)}-\beta_{i}$ 's $(i=1,2, \ldots, n)$ are the distinct simple factors of $G-\frac{B-A}{B}$. Clearly from above we get

$$
\sum_{i=1}^{n} \bar{N}\left(r, \beta_{i} ; g^{(k)}\right)=\bar{N}(r, \infty ; f)
$$

Again by the second fundamental theorem we get

$$
\begin{aligned}
(n-1) T\left(r, g^{(k)}\right) & \leq \sum_{i=1}^{n} \bar{N}\left(r, \beta_{i} ; g^{(k)}\right)+\bar{N}(r, \infty ; g)+S\left(r, g^{(k)}\right) \\
& \leq \bar{N}(r, \infty ; f)+\bar{N}(r, \infty ; g)+S\left(r, g^{(k)}\right)
\end{aligned}
$$

i.e., in view of $(3.2)$

$$
\left(n-1-\frac{2}{k+1}\right) T\left(r, g^{(k)}\right) \leq S\left(r, g^{(k)}\right),
$$

which is a contradiction for $n \geq 3$.

Subcase 1.2.2.2.

If $A-B=0$, then from (3.3) we get

$$
\frac{B}{-b}\left(g^{(k)}\right)^{n-1}\left(g^{(k)}+a\right) \equiv \frac{G-1}{F-1} .
$$


Using the similar argument as in Subcase 1.2.2.1.1. we get that zeros and ' $-a$ ' points of $g^{(k)}$ are nutralised by the poles of $f$. Also we have $f^{(k)}, g^{(k)}$ share $(0,0)$ therefore from the above equation we get that 0 is an e.v.P. of $g^{(k)}$ and

$$
\bar{N}\left(r,-a ; g^{(k)}\right) \leq \frac{1}{n(k+1)} T\left(r, f^{(k)}\right) .
$$

So by the second fundamental theorem and (3.2) we get

$$
\begin{aligned}
T\left(r, g^{(k)}\right) & \leq \bar{N}\left(r,-a ; g^{(k)}\right)+\bar{N}\left(r, 0 ; g^{(k)}\right)+\bar{N}(r, \infty ; g)+S\left(r, g^{(k)}\right) \\
& \leq\left\{\frac{1}{n(k+1)}+\frac{1}{k+1}\right\} T\left(r, g^{(k)}\right)+S\left(r, g^{(k)}\right),
\end{aligned}
$$

a contradiction for $n \geq 3$.

Case 2. Suppose that $\Phi \equiv 0$. On integration we get

$$
(F-1) \equiv A(G-1)
$$

for some non-zero constant $A$. Here also in view of Lemma 2.4, (3.2) holds. Since by the given condition of the theorem $E_{f}\left(S_{1}, 0\right)=E_{g}\left(S_{1}, 0\right)$ we consider the following subcases.

Subcase 2.1. Suppose $A \neq 1$ then from (3.5) we get

$$
\frac{F}{A} \equiv G+\frac{1-A}{A}
$$

Now let us consider the following subcases.

Subcase 2.1.1. Suppose $G+\frac{1-A}{A}$ has $n-2$ distinct zeros, $\eta_{i}, i=1,2, \ldots, n-2$ and a double zero at $c_{1}$. Then from (3.6) we get

$$
\frac{\left(f^{(k)}\right)^{n-1}\left(f^{(k)}+a\right)}{A} \equiv\left(g^{(k)}-c_{1}\right)^{2}\left(g^{(k)}-\eta_{1}\right)\left(g^{(k)}-\eta_{2}\right) \ldots\left(g^{(k)}-\eta_{n-2}\right) .
$$

Since $f^{(k)}, g^{(k)}$ share $(0,0)$, then from (3.7) ' 0 ' is clearly an e.v.P of $f^{(k)}$ and hence e.v.P. of $g^{(k)}$. So again from the second fundamental theorem we get

$$
\begin{aligned}
& (n-1) T\left(r, g^{(k)}\right) \\
\leq & \sum_{i=1}^{n-2} \bar{N}\left(r, \eta_{i} ; g^{(k)}\right)+\bar{N}\left(r, c_{1} ; g^{(k)}\right)+\bar{N}\left(r, 0 ; g^{(k)}\right)+\bar{N}(r, \infty ; g)+S\left(r, g^{(k)}\right) \\
\leq & \bar{N}\left(r,-a ; f^{(k)}\right)+\frac{1}{k+1} T\left(r, g^{(k)}\right)+S\left(r, g^{(k)}\right),
\end{aligned}
$$

which in view of (3.2) gives a contradiction for $n \geq 3$.

Subcase 2.1.2 Suppose $G+\frac{1-A}{A}$ has $n$ distinct zeros, $\xi_{i}, i=1,2, \ldots, n$. Then (3.5) takes the form

$$
\frac{\left(f^{(k)}\right)^{n-1}\left(f^{(k)}+a\right)}{A} \equiv\left(g^{(k)}-\xi_{1}\right)\left(g^{(k)}-\xi_{2}\right) \ldots\left(g^{(k)}-\xi_{n}\right) .
$$


Similarly as above we can prove here that ' 0 ' is an e.v.P. of $g^{(k)}$. Then from the second fundamental theorem we get

$$
\begin{aligned}
& n T\left(r, g^{(k)}\right) \\
\leq & \sum_{i=1}^{n} \bar{N}\left(r, \xi_{i} ; g^{(k)}\right)+\bar{N}\left(r, 0 ; g^{(k)}\right)+\bar{N}\left(r, \infty ; g^{(k)}\right)+S\left(r, g^{(k)}\right) \\
\leq & \bar{N}\left(r,-a ; f^{(k)}\right)+\frac{1}{k+1} T\left(r, g^{(k)}\right)+S\left(r, g^{(k)}\right),
\end{aligned}
$$

which in view of (3.2) gives a contradiction for $n \geq 3$.

Subcase 2.2. Suppose $A=1$ then we have $F \equiv G$, which in view of Lemma 2.7 implies $f^{(k)} \equiv g^{(k)}$.

\section{Concluding remark and an open question}

Theorem 1.1 shows that all the zeros of the derivatives of the underlying polynomial is not necessary to form $S_{1}$. Also Example 1.2 shows that $S_{2}$ of Theorem 1.1 cannot be replaced by any arbitrary set containing 4 elements. Using the method adopted to prove Theorem 1.1 one can verify that for any underlying polynomial of a BURSM the lower bound of the degree of the polynomial cannot be reduced further. Therefore the following question is ineludible for the construction of BURSM.

Question 4.1. Does there exist any pair of Bi-Unique range sets, even if for a special class of meromorphic functions, sum of whose cardinalities are less than 5 ?

\section{Acknowledgement}

This research work is supported by the Council Of Scientific and Industrial Research, Extramural Research Division, CSIR Complex, Pusa, New Delhi-110012, India, under the sanction project no. 25(0229)/14/EMR-II.

\section{References}

[1] A. Banerjee, On the uniqueness of meromorphic functions that share two sets, Georgian Math., 15 (1) (2008), 21-38.

[2] A. Banerjee, Bi-unique range sets for meromorphic functions, Nihonkai Math. J., 24(2) (2013), $121-134$

[3] A. Banerjee and P. Bhattacharajee, Uniqueness of derivatives of meromorphic function sharing two or three sets, Turkish J. Math., 34(1) (2010), 21-34.

[4] A. Banerjee and P. Bhattacharajee, Uniqueness and set sharing of derivatives of meromorphic functions, Math. Slovaca, 61(2) (2010), 197-214.

[5] A. Banerjee and S. Mallick, Uniqueness of meromorphic functions sharing two finite sets in $\mathbb{C}$ with finite weight II, Rend. Circ. Mat. Palermo., DOI 10.1007/s12215-015-0208-8. 
[6] M. Fang and H. Guo, On meromorphic functions sharing two values, Analysis 17 (1997), 355366.

[7] F. Gross, Factorization of meromorphic functions and some open problems, Proc. Conf. Univ. Kentucky, Leixngton, Ky(1976); Lecture Notes in Math., 599 (1977), 51-69, Springer(Berlin).

[8] W. K. Hayman, Meromorphic functions, The Clarendon Press, Oxford (1964).

[9] I. Lahiri, Value distribution of certain differential polynomials, Int. J. Math. Math. Sci., 28(2) (2001), 83-91.

[10] I. Lahiri, Weighted sharing and uniqueness of meromorphic functions, Nagoya Math. J., 161 (2001), 193-206.

[11] I. Lahiri, Weighted value sharing and uniqueness of meromorphic functions, Complex Var. Theory Appl., 46 (2001), 241-253.

[12] I. Lahiri, On a question of Hong Xun Yi, Arch. Math. (Brno), 38 (2002), 119-128.

[13] I. Lahiri and A. Banerjee, Uniqueness of meromorphic functions with deficient poles, Kyungpook Math. J., 44 (2004), 575-584.

[14] P. Li and C. C. Yang, On the unique range set for meromorphic functions, Proc. Amer. Math. Soc., 124 (1996), 177-185.

[15] A. Z. Mokhon'ko, On the Nevanlinna characteristics of some meromorphic functions, in "Theory of functions, functional analysis and their applications", Izd-vo Khar'kovsk, Un-ta, 14 (1971), 83-87.

[16] B. Yi and Y. H. Li, The uniqueness of meromorphic functions that share two sets with CM, Acta Math. Sinica Chinese Ser., 55(2) (2012), 363-368.

[17] H. X. Yi, Uniqueness of meromorphic functions and a question of Gross, Sci. China (A), 37(7) (1994), 802-813.

[18] H. X. Yi, Meromorphic functions that share two sets, Acta Math Sinica, 45 (2002), 75-82

[19] H. X. Yi and W. C. Lin, Uniqueness of meromorphic functions and a question of Gross, Kyungpook Math. J., 46 (2006), 437-444. 